\title{
Nonspecific interstitial pneumonia in refractory systemic juvenile idiopathic arthritis responded to tocilizumab treatment
}

\author{
Maynart Sukharomana, ${ }^{1}$ Kanokporn Udomittipong, ${ }^{2}$ Ruchira Ruangchira-urai, ${ }^{3}$ Sirirat Charuvanij $^{1}$
}

\begin{abstract}
Background: Nonspecific interstitial pneumonia (NSIP) is a rare pulmonary complication in systemic juvenile idiopathic arthritis (SJIA).

Objective: To present a case with NSIP in SJIA

Methods: Case report

Results: We report the case of a 4-year-old boy with SJIA complicated by macrophage activation syndrome (MAS) refractory to conventional therapy, and who later developed NSIP confirmed by high-resolution computerized tomography of the chest and lung histopathology. The patient received tocilizumab, a monoclonal antibody to interleukin- 6 receptor, to control his disease. Data relating to tocilizumab treatment of NSIP in refractory SJIA is limited.
\end{abstract}

Conclusions: The data from this case report suggests that NSIP could be a pulmonary complication of SJIA complicated by MAS that is refractory to conventional therapy. Early initiation of tocilizumab should be considered to achieve disease remission in this pediatric patient population.

Key words: macrophage activation syndrome, nonspecific interstitial pneumonia, refractory systemic juvenile idiopathic arthritis, tocilizumab, interleukin-6

\section{Citation:}

Sukharomana, M., Udomittipong, K., Ruangchira-urai, R., Charuvanij, S. (2022). Nonspecific interstitial pneumonia in refractory systemic juvenile idiopathic arthritis responded to tocilizumab treatment. Asian Pac J Allergy Immunol, 40(2), 173-177. https:// doi.org/10.12932/ap-050819-0616

\footnotetext{
Affiliations:

Division of Rheumatology, Department of Pediatrics, Faculty of Medicine Siriraj Hospital, Mahidol University, Bangkok, Thailand

${ }^{2}$ Division of Pulmonary Medicine, Department of Pediatrics, Faculty of Medicine Siriraj Hospital, Mahidol University, Bangkok, Thailand

${ }^{3}$ Department of Pathology, Faculty of Medicine Siriraj Hospital, Mahidol University, Bangkok, Thailand
}

Corresponding author:

Sirirat Charuvanij

Division of Rheumatology, Department of Pediatrics

Faculty of Medicine Siriraj Hospital, Mahidol University

2 Wanglang Road, Bangkoknoi, Bangkok 10700, Thailand

E-mail: sirirat.chv@mahidol.ac.th

\section{Introduction}

Juvenile idiopathic arthritis (JIA) comprises a group of diseases defined according to the following International League of Associations for Rheumatology (ILAR) classification criteria: onset occurs before the age of 16 years with a duration of arthritis of at least 6 weeks, excluding other conditions. ${ }^{1}$ Systemic juvenile idiopathic arthritis (SIA), one of the 7 subtypes of JIA with distinct clinical presentation and complication, is defined as arthritis with or preceded by fever for at least 2 weeks with quotidian pattern for at least 3 consecutive days accompanied by evanescent erythematous rash, generalized lymphadenopathy, hepatomegaly and/or splenomegaly, and serositis. ${ }^{1}$ In patients with onset of symptoms at age 16 years or older, adult-onset Still's disease (AOSD) which could be considered a spectrum of SIIA, can be classified.

The pathogenesis of SJIA is attributable to dysregulation of the innate immune system, with more autoinflammatory predominance compared to the other JIA subtypes. ${ }^{2}$ Patients with SJIA are also at risk for developing macrophage activation syndrome (MAS), a potentially fatal complication. Abnormal cytolytic pathway, decreased natural killer cell activities, 
and the hyperinflammatory state of SJIA disease result in prolonged immune activation, especially the activation of cytotoxic $\mathrm{T}$ cells and macrophages. ${ }^{3}$ Involved cytokines include interferon gamma, interleukin (IL)-1, IL-2, IL-6, IL-18, tumor necrosis factor-alpha, and macrophage colony-stimulating factor. $^{2-4}$ Patients with SJIA complicated with MAS usually present with non-remitting fever, cytopenias, liver dysfunction, coagulopathy, and central nervous system dysfunction, which highlights the need for early suspicion, prompt diagnosis, and appropriate treatment to prevent mortality. ${ }^{2}$

Pulmonary manifestations, except for serositis, are rare in SJIA. Reported findings in patients with SJIA include pulmonary arterial hypertension (PAH), interstitial lung disease (ILD), and alveolar proteinosis. ${ }^{5}$ ILD in childhood is a group of chronic respiratory disorders with inflammatory and fibrotic changes that adversely affect alveolar walls. Non-specific interstitial pneumonia (NSIP), which is one of the ILD subgroups, is characterized by chronic interstitial inflammation with type 2 alveolar epithelial cell hyperplasia at areas of inflammation. Data relating to the treatment of ILD in SJIA and the use of tocilizumab to treat this complication is limited. Here, we report the case of a 4-year-old Thai boy with NSIP in refractory SJIA complicated by MAS who responded to tocilizumab treatment.

\section{Case presentation}

A 4-year-old Thai boy diagnosed as SJIA complicated with MAS initially presented with prolonged quotidian fever, evanescent erythematous rash, generalized lymphadenopathy, and serositis. Chest examination showed respiratory rate 42/ minute, subcostal retraction, normal breath sounds, and no adventitious sounds. Chest X-ray showed mild cardiomegaly without lung infiltration. Serum ferritin level was 343,299 $\mathrm{ng} / \mathrm{ml}$, and bone marrow aspiration showed hemophagocytic activity. He was treated by 3 doses of pulse methylprednisolone (30 mg/kg/dose), intravenous immunoglobulin (IVIG), cyclosporine, and oral prednisolone. However, during the first 6 months, he still had some episodes of quotidian fever,
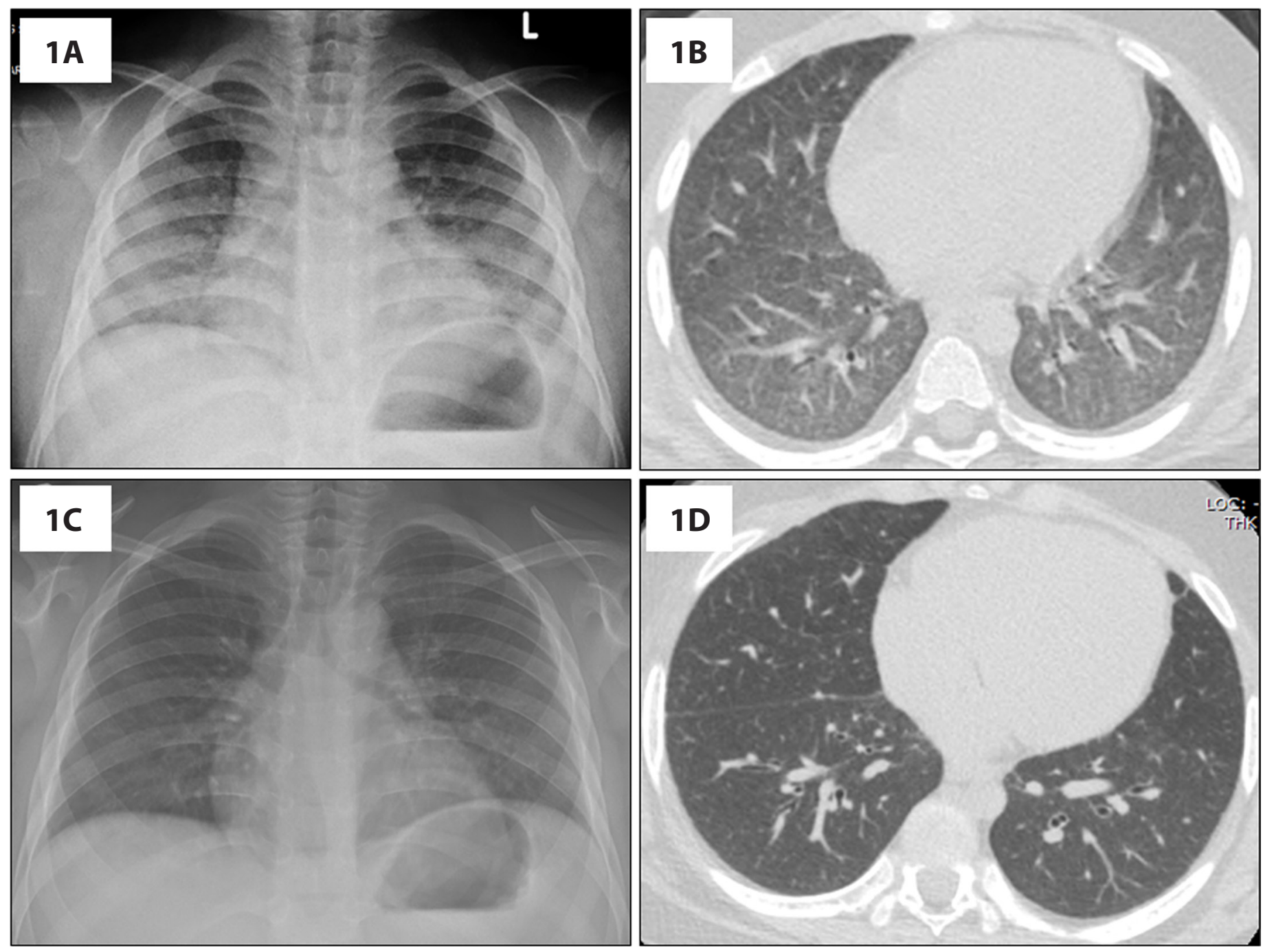

Figure 1. (A) Chest X-ray showed bilateral interstitial infiltration with peribronchial thickening. (B) HRCT of the chest showed interlobular septal thickening with peripheral ground glass opacities at the posterior aspect of both lungs. (C) Chest $\mathrm{X}$-ray at 2 years of follow-up showed decreased interstitial infiltration. (D) HRCT chest showed decreased degree of diffuse interlobular septal thickening and reticular opacity in both lungs. 
evanescent erythematous rash, arthritis despite receiving monthly pulse methylprednisolone, and therefore could not achieve clinical remission. Seven months after diagnosis he developed dyspnea, tachypnea concurrent with fever, evanescent erythematous rash, and arthritis. Vital signs showed temperature 39 degrees Celsius, blood pressure 119/56 $\mathrm{mmHg}$, respiratory rate $40 /$ minute, and pulse oximetry $97 \%$ in room air. Chest examination showed retraction and crepitations at both lungs. Chest X-ray revealed bilateral interstitial infiltration with peribronchial thickening (Figure 1A). Blood tests to reevaluate the SJIA disease activity were performed and were not suggestive of MAS. Echocardiogram showed no pericardial effusion, intracardiac shunt, or evidence suggestive of pulmonary arterial hypertension (PAH) (right ventricular systolic pressure $35 \mathrm{mmHg}$, less than $50 \%$ of systolic blood pressure) with good ventricular function (ejection fraction 59\%). He was initially treated as pneumonia by cefotaxime and azithromycin. Investigations for infectious etiologies showed negative results, including hemoculture, blood $M y$ coplasma titer, gastric washes for Mycobacterium tuberculosis, and nasopharyngeal wash for respiratory viruses. Bronchoscopy revealed swelling of bronchi with erythema of mucosa.
Bronchoalveolar lavage showed 155 cells/ml (neutrophils $71 \%$, lymphocytes $4 \%$, monocytes $2 \%$, eosinophils $1 \%$, and macrophages 22\%), with negative stains, PCR, and/or cultures for Pneumocystis jirovecii, respiratory viruses, mycobacteria, fungus, and bacteria. High-resolution computerized tomography (HRCT) chest showed interlobular septal thickening with peripheral ground glass opacities at the posterior aspect of both lungs (Figure 1B). Surgical lung biopsy pathology revealed diffuse interstitial thickening with chronic inflammatory cell infiltration and fibrosis, diffuse alveolar cell hyperplasia, intraalveolar accumulation of foamy alveolar macrophages, and cholesterol clefts (Figures 2A-D). Honeycomb fibrosis was not identified. No granuloma, viral cytopathic change, or vasculitis was identified. Special stains were also negative for acid-fast bacilli and fungus. All lung histopathology findings were consistent with non-specific interstitial pneumonia (NSIP) pattern. The serum IL-6 level was $418 \mathrm{pg} / \mathrm{ml}$. Tocilizumab, a monoclonal antibody to IL-6 receptor, was administrated intravenously (12 mg/kg/dose) for every 2 weeks in combination with systemic corticosteroids in the form of methylprednisolone $30 \mathrm{mg} / \mathrm{kg} / \mathrm{dose}$ for 3 days with subsequent prednisolone tapering, and oral cyclosporine
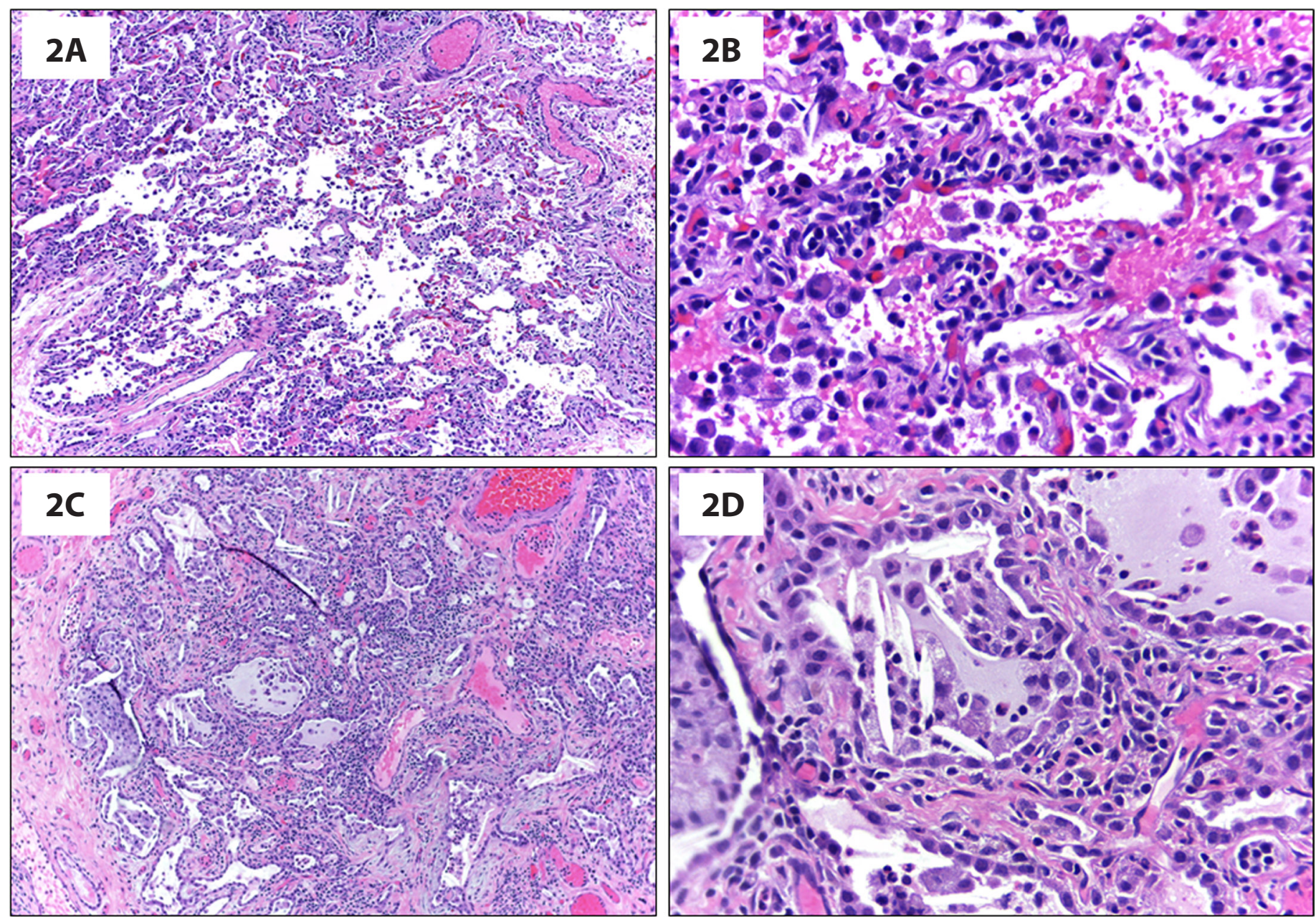

Figure 2. (A) Diffuse interstitial thickening due to chronic inflammatory cell infiltrate with some intraalveolar macrophages (H\&E staining, 100× magnification). (B) Interstitial cell infiltrate consisting mainly of small lymphocytes (H\&E staining, 400× magnification). (C) More prominent interstitial inflammation and fibrosis with intraalveolar mucus, histiocytes, and cholesterol clefts (H\&E staining, 100× magnification). (D) Alveolar spaces lined by hyperplastic type 2 pneumocytes containing mucus, foamy macrophages, and cholesterol clefts (H\&E staining, 400× magnification). 
(4 mg/kg/day). His SJIA and NSIP gradually improved as evidenced by the improvement of clinical signs andsymptoms of systemic features and respiratory distress at 6 months. The interval of tocilizumab was subsequently extended to every 4 weeks. At the 2-year imaging follow-up, the chest X-ray showed decreased interstitial infiltration (Figure 1C) comparing to the film at the diagnosis of NSIP (Figure 1A) and the HRCT chest showed decreased degree of diffuse interlobular septal thickening and reticular opacity in both lungs (Figure 1D). Bronchoalveolar lavage and biopsy was not repeated. At that time, the patient's disease status was inactive while still receiving tocilizumab every 4 weeks along with tapering of prednisolone to $7.5 \mathrm{mg}$ /day and discontinuation of cyclosporine.

\section{Discussion}

Except for serositis, pulmonary complications in SJIA are rare. The pulmonary complications that can develop include PAH (64\%), ILD (28\%), and alveolar proteinosis (20\%). ${ }^{5} \mathrm{~Pa}-$ tients with pulmonary complications had more systemic manifestations and more previous treatment for SJIA disease. ${ }^{5}$ Pulmonary conditions may be the result of severe uncontrolled active systemic disease or may be influenced by exposure to medications. ${ }^{5}$

Data relating to SJIA with ILD and treatment are scarce. Earlier case series and review reported pulmonary involvement in juvenile rheumatoid arthritis (JRA). ${ }^{6,7}$ A previous report profiled a girl with JRA who had parenchymal lung disease, joint symptoms, and subcutaneous nodules compatible with seropositive polyarticular-onset JRA. ${ }^{6}$ Another report profiled a girl with refractory systemic chronic rheumatoid arthritis who developed pulmonary interstitial and intra-alveolar cholesterol granulomas three years later. She was clinically improved by etanercept, but later died of respiratory insufficiency. ${ }^{7}$ Radiographic findings of ILD included transient pneumonitis, interstitial infiltration, pleural effusions, and patchy pleural infiltration. Pathologic findings showed pulmonary hemosiderosis, lymphoid follicular bronchiolitis, and lymphocytic interstitial pneumonitis. ${ }^{8}$ Cholesterol clefts are caused by deposition and organization of cholesterol released from degenerating lipid laden macrophages. ${ }^{9}$

There are also reports of AOSD with pulmonary involvement in adults. One study reported parenchymal lung involvement in $5.3 \%$ of AOSD cases. ${ }^{10}$ In cases without acute respiratory distress syndrome, HRCT chest showed interstitial hyperdensities, including NSIP, organizing pneumonia, and unclassified ILD. Biopsy-proven NSIP was found in 2 cases. All cases with NSIP in AOSD were treated with systemic corticosteroids without biologic agents. ${ }^{10} \mathrm{~A}$ case report of a patient with AOSD with PAH and NSIP complicated by MAS showed total resolution of MAS and improvement of PAH after treatment by systemic corticosteroids, cyclosporine A, and anakinra. ${ }^{11}$ Taken together, data specific to tocilizumab treatment for SJIA or AOSD with NSIP is limited. In contrast, ILD are known to be a pulmonary complication of rheumatoid arthritis (RA). Tocilizumab was reported to exacerbate ILD or worsen lung fibrosis in adults with RA. ${ }^{12,13}$ Nevertheless, there were reports of RA patients with associated ILD successfully treated by tocilizumab as monotherapy. ${ }^{14,15}$
Regarding pediatric populations, tocilizumab is effective in treating SJIA, particularly those with severe or persistent diseases, ${ }^{16,17}$ with one study including 6 of 32 patients with ILD (16.2\%). ${ }^{17}$ A large study in children with SJIA using tocilizumab did not report ILD-related adverse effects. ${ }^{18}$ IL-6, one of the cytokines which plays a major role in pathogenesis of SJIA, could be found elevated in the blood and synovial fluid in patients with active disease, with levels correlating with disease activity. ${ }^{19}$ In our patient, given that the ongoing active systemic features with NSIP and the IL-6 level was very high, tocilizumab was considered for treatment with prednisolone and cyclosporine. After 2 years, the response to treatment of SJIA and NSIP therefore could be the effect of tocilizumab as well as the concurrent immunosuppressive drugs. Further studies of tocilizumab as monotherapy of NSIP in patients with SJIA should be warranted. Moreover, there could be potential genetic polymorphisms such as the STAT3 gain-offunction which play important roles in regulation of inflammatory cytokines including IL-6, and STAT3 gain-of-function that might be associated with NSIP in SJIA patients, as it has been reported in patients with interstitial lung diseases. ${ }^{20}$ However, the genetic testing was not performed in this patient to confirm this postulation.

In conclusion, the case profiled in this report highlights that children with refractory SJIA complicated with MAS at the time of diagnosis could later develop NSIP and that early administration of tocilizumab could be beneficial.

\section{Acknowledgments}

The authors gratefully acknowledge the patient profiled in this study and his parents for giving us written informed consent to report details relating to his case.

\section{Conflict of interest declaration}

All authors hereby declare no personal or professional conflicts of interest, and no financial support from the companies that produce and/or distribute the drugs, devices, or materials described in this report.

\section{Funding disclosure}

This was an unfunded study.

\section{References}

1. Petty RE, Southwood TR, Manners P, Baum J, Glass DN, Goldenberg J, et al. International League of Associations for Rheumatology classification of juvenile idiopathic arthritis: second revision, Edmonton, 2001. J Rheumatol. 2004;31:390-2.

2. Yasin S, Schulert GS. Systemic juvenile idiopathic arthritis and macrophage activation syndrome: update on pathogenesis and treatment. Curr Opin Rheumatol. 2018;30:514-20.

3. Lerkvaleekul B, Vilaiyuk S. Macrophage activation syndrome: early diagnosis is key. Open Access Rheumatol. 2018;10:117-28.

4. Pardeo M, Bracaglia C, De Benedetti F. Systemic juvenile idiopathic arthritis: New insights into pathogenesis and cytokine directed therapies. Best Pract Res Clin Rheumatol. 2017;31:505-16.

5. Kimura Y, Weiss JE, Haroldson KL, Lee T, Punaro M, Oliveira S, et al. Pulmonary hypertension and other potentially fatal pulmonary complications in systemic juvenile idiopathic arthritis. Arthritis Care Res (Hoboken). 2013;65:745-52. 
6. Noyes BE, Albers GM, deMello DE, Rubin BK, Moore TL. Early onset of pulmonary parenchymal disease associated with juvenile rheumatoid arthritis. Pediatr Pulmonol. 1997;24:444-6.

7. Schultz R, Mattila J, Gappa M, Verronen P. Development of progressive pulmonary interstitial and intra-alveolar cholesterol granulomas (PICG) associated with therapy-resistant chronic systemic juvenile arthritis (CJA). Pediatr Pulmonol. 2001;32:397-402.

8. Athreya BH, Doughty RA, Bookspan M, Schumacher HR, Sewell EM, Chatten J. Pulmonary manifestations of juvenile rheumatoid arthritis. A report of eight cases and review. Clin Chest Med. 1980;1:361-74.

9. Fisher M, Roggli V, Merten D, Mulvihill D, Spock A. Coexisting endogenous lipoid pneumonia, cholesterol granulomas, and pulmonary alveolar proteinosis in a pediatric population: a clinical, radiographic, and pathologic correlation. Pediatr Pathol. 1992;12:365-83.

10. Gerfaud-Valentin M, Cottin V, Jamilloux Y, Hot A, Gaillard-Coadon A, Durieu I, et al. Parenchymal lung involvement in adult-onset Still disease: A STROBE-compliant case series and literature review. Medicine (Baltimore). 2016;95:e4258.

11. Mehta MV, Manson DK, Horn EM, Haythe J. An atypical presentation of adult-onset Still's disease complicated by pulmonary hypertension and macrophage activation syndrome treated with immunosuppression: a case-based review of the literature. Pulm Circ. 2016;6:136-42.

12. Kawashiri SY, Kawakami A, Sakamoto N, Ishimatsu Y, Eguchi K. A fatal case of acute exacerbation of interstitial lung disease in a patient with rheumatoid arthritis during treatment with tocilizumab. Rheumatol Int. 2012;32:4023-6.
13. Le TT, Karmouty-Quintana H, Melicoff E, Le TT, Weng T, Chen NY, et al. Blockade of IL-6 Trans signaling attenuates pulmonary fibrosis. J Immunol. 2014;193:3755-68.

14. Picchianti Diamanti A, Markovic M, Argento G, Giovagnoli S, Ricci A, Lagana B, et al. Therapeutic management of patients with rheumatoid arthritis and associated interstitial lung disease: case report and literature review. Ther Adv Respir Dis. 2017;11:64-72.

15. Mohr M, Jacobi AM. Interstitial lung disease in rheumatoid arthritis: response to IL-6R blockade. Scand J Rheumatol. 2011;40:400-1.

16. De Benedetti F, Brunner HI, Ruperto N, Kenwright A, Wright S, Calvo I, et al. Randomized trial of tocilizumab in systemic juvenile idiopathic arthritis. N Engl J Med. 2012;367:2385-95.

17. Kostik MM, Dubko MF, Masalova VV, Snegireva LS, Kornishina TL, Chikova IA, et al. Successful treatment with tocilizumab every 4 weeks of a low disease activity group who achieve a drug-free remission in patients with systemic-onset juvenile idiopathic arthritis. Pediatr Rheumatol Online J. 2015;13:4.

18. Yokota S, Itoh Y, Morio T, Origasa H, Sumitomo N, Tomobe M, et al. Tocilizumab in systemic juvenile idiopathic arthritis in a real-world clinical setting: results from 1 year of postmarketing surveillance follow-up of 417 patients in Japan. Ann Rheum Dis. 2016;75:1654-60.

19. Lerkvaleekul B, Vilaiyuk S. Early reduction of serum interleukin-6 levels as a predictor of clinical remission in systemic juvenile idiopathic arthritis. Asian Pac J Allergy Immunol. 2019;37:116-22.

20. Fabre A, Marchal S, Forbes LR, Vogel TP, Barlogis V, Triolo V, et al. STAT3 Gain of Function: A New Kid on the Block in Interstitial Lung Diseases. Am J Respir Crit Care Med. 2018;197:e22-3. 